\title{
ECOCARDIOGRAFIA MODO M EM CÃES NORMAIS DA RAÇA PASTOR ALEMÃO (ORIGEM AMERICANA) DO CANIL DA POLÍCIA MILITAR DO ESTADO DE MINAS GERAIS, BRASIL
}

\author{
M-MODE ECHOCARDIOGRAPHY IN NORMAL GERMAN SHEPHERD DOGS (AMERICAM \\ ORIGIN) FROM MINAS GERAIS STATE POLICE KENNEL, BRAZIL
}

\author{
Ruthnéa Aparecida Lázaro Muzzi ${ }^{1}$, Roberto Baracat de Araújo², Leonardo Augusto Lopes Muzzi ${ }^{3}$, \\ José Luiz Barros Pena ${ }^{4}$
}

\section{RESUMO}

Este estudo teve como objetivo estabelecer os índices ecocardiográficos modo- $M$ normais de cães da raça Pastor Alemão do canil da Polícia Militar do Estado de Minas Gerais. Sessenta cães clinicamente sadios foram submetidos ao exame ecocardiográfico. $O$ diâmetro interno do ventrículo direito na diástole e do ventrículo esquerdo na diástole e na sístole, massa ventricular esquerda, dimensão do arco aórtico e átrio esquerdo, espessura do septo interventricular e parede posterior do ventrículo esquerdo na diástole e sístole tiveram correlações significativas com o peso corporal. A relação espessura do septo interventricular e parede posterior, no final da diástole, relação diâmetro do átrio esquerdo e aorta, porcentagem de espessamento sistólico do septo interventricular, porcentagem de espessamento sistólico da parede posterior de ventrículo esquerdo e porcentagem de encurtamento sistólico do diâmetro ventricular esquerdo não se correlacionaram com o peso corporal, sexo ou idade.

Palavras-chave: ecocardiografia Modo-M, coração, cão.

SUMMARY

The normal M-mode echocardiographic parameters were determined in German Shepherd dogs from Minas Gerais State Police kennel. Sixty clinically normal dogs were submitted to the echocardiographic examination. The right ventricular internal dimension at end-diastole, the left ventricular internal dimension at end-diastole and end-systole, the left ventricular mass, the aortic root dimension, left atrial systolic dimension, end-diastolic interventricular septal thickness and end-diastolic and end-systolic left ventricular posterior wall thickness had significant correlation with body weight. The ratio of septal to left ventricular posterior wall thickness at end-distole, the left atrial to aortic root ratio, the thickening fraction of the interventricular septum, the thickening fraction of the left ventricular posterior wall, and left ventricular shortening fraction showed no linear correlations with body weight, sex or age.

Key words: M-mode echocardiography, heart, dog

\section{INTRODUÇÃO}

A ecocardiografia é a técnica de utilização do ultra-som como meio diagnóstico em cardiologia (MORCERF, 1996), que vem sendo usada na medicina veterinária nos últimos anos, representando um importante método não-invasivo na avaliação morfofuncional do coração (HENIK, 1995; DROUARDHAELEWYN, 1998).

No modo bidimensional, conhecido como ecocardiografia em tempo real, a imagem obtida é a de um plano de corte, reconstituindo, em duas dimensões, as estruturas anatômicas estudadas (JAUDON et al., 1991). O modo-M exibe, por meio de um gráfico, sinais de diferentes ecogenicidades devido à profundidade dos tecidos, registrando o movimento do coração através de uma linha de tempo (HENIK, 1995; MARTIN, 1995). É obtido através da colocação da linha do cursor sobre a área que

\footnotetext{
${ }^{1}$ Médico Veterinário, MSc., Professor Assistente, Departamento de Medicina Veterinária, Campus Universitário da Universidade Federal de Lavras (UFLA), Caixa Postal 37, 37200-000, Lavras, MG. E-mail: ralmuzzi@ufla.br. Autor para correspondência.

${ }^{2}$ Médico Veterinário, Doutor, Professor Adjunto, Escola de Veterinária da Universidade Federal de Minas Gerais.

${ }^{3}$ Médico Veterinário, MSc., Professor Assistente, Departamento de Medicina Veterinária, UFLA.

${ }^{4}$ Médico, MSc., Hospital Felício Rocho.
} 
se deseja pesquisar na imagem bidimensional do coração. O gráfico resultante será demonstrado em função do tempo, onde as estruturas que não se movimentam são apresentadas como linhas retas, e aquelas que têm movimentos aparecem como linhas onduladas (BONAGURA, 1994). Pelo modo-M é possível quantificar a dimensão das câmaras cardíacas, grandes vasos, espessura das paredes, movimento valvular e os índices funcionais do ventrículo esquerdo, que são especialmente importantes para avaliar as diversas alterações no coração. $\mathrm{O}$ ventrículo esquerdo e direito, átrio esquerdo, aorta e valva mitral são, freqüentemente, estudados no modo-M (ALLEN, 1982; HENIK, 1995; KIENLE \& THOMAS, 1995)

Segundo BOON et al. (1983), LOMBARD (1984), GOODING et al. (1986), JACOBS \& MAHJOOB (1988), KIRBERGER (1991A), SISSON \& SCHAEFFER (1991) e KOCH et al. (1996), a imagem do modo bidimensional ideal para se colocar a linha do cursor do modo-M para estudar o ventrículo esquerdo e direito é a visão paraesternal direita transversal, nas cordoalhas tendíneas. Essa padronização é necessária para que se possam comparar os parâmetros ecocardiográficos entre pacientes diferentes ou mesmo em exames seriados de um mesmo animal.

Os índices ecocardiográficos apresentam grande variabilidade racial e, tendo em vista a influência do peso corporal, sexo e idade nas mensurações ecocardiográficas preestabelecidas, torna-se necessário conhecer os valores ecocardiográficos normais para cada raça (BOON et al., 1983; CRIPPA et al., 1992 e SNYDER et al., 1995). Dessa forma, o objetivo deste trabalho é determinar os índices ecocardiográficos do modo $\mathrm{M}$ no cão Pastor Alemão, clinicamente sadio, e correlacioná-los com peso corporal, sexo e idade, uma vez que não se têm estudos a esse respeito. Os dados obtidos poderão contribuir para o avanço dos estudos na clínica médica veterinária, mormente na área da cardiologia em pequenos animais.

\section{MATERIAL E MÉTODOS}

Foram utilizados 60 cães da raça Pastor Alemão, clinicamente sadios, 31 machos e 29 fêmeas, com idade variando entre um e cinco anos, pertencentes ao canil da Polícia Militar do Estado de Minas Gerais, Brasil.

Os cães foram selecionados por meio de exame físico e eletrocardiográfico, sendo excluídos os que apresentassem qualquer sinal clínico de cardiopatia. Foram submetidos ao exame ecocardiográfico, modos bidimensional e M, sendo as observa- ções anotadas em fichas próprias e individuais, que continham dados, como nome do animal, número de ordem, idade, sexo e peso corporal.

Para a realização do exame ecocardiográfico, foi utilizado um aparelho de ultra-sonografia ${ }^{a}$, composto por modos bidimensional e $\mathrm{M}$, com transdutor de $3,5 \mathrm{mHz}$ e acompanhamento eletrocardiográfico no monitor, fornecendo a freqüência cardíaca. Os animais foram posicionados em decúbito lateral direito, por meio de contenção manual, não sendo necessário o uso de sedação. Foi utilizada uma mesa com abertura em forma de U na altura da região torácica para facilitar a colocação do transdutor no sítio do exame.

Uma espessa camada de gel acústico ${ }^{b}$ foi usado entre o transdutor e o local do exame, não sendo necessária a tricotomia da região. Foram realizadas três medidas de cada variável, individualmente, e obtidas médias dos dados. Todos os exames foram impressos ${ }^{c}$ em papel termossensível ${ }^{\mathrm{d}}$ e gravados em videoteipe ${ }^{\mathrm{e}}$ para posteriores avaliações.

Para obtenção das imagens do modo bidimensional, utilizou-se a região paraesternal direita, do terceiro ao quinto espaços intercostais (janelas acústicas), seguindo-se as recomendações de THOMAS et al. (1993). Por meio desse modo foi obtido o modo M, seguindo-se recomendações de SAHN et al. (1978) e LOMBARD (1984). Ao final da diástole, foram obtidas as medidas: diâmetro da raiz da aorta (AO); dimensão interna dos ventrículos direito (VDd) e esquerdo (VEd); espessuras do septo interventricular (SIVd) e da parede posterior do ventrículo esquerdo (PPd). Medidas realizadas no final da sístole: diâmetro interno do átrio esquerdo (AE); dimensão interna do ventrículo esquerdo (VEs); espessuras do septo interventricular (SIVs) e da parede posterior do ventrículo esquerdo (PPs).

Após a obtenção das medidas citadas acima, foram calculados, segundo BOON et al. (1983): relação espessura do septo interventricular e parede posterior, no final da diástole (SIV/PP), relação diâmetro do átrio esquerdo e aorta (AEs/AOd), porcentagem de encurtamento sistólico do diâmetro ventricular esquerdo $(\% \Delta \mathrm{d})$, porcentagem de espessamento sistólico do septo interventricular (\%ESS) e da parede posterior do ventrículo esquerdo (\%EPP).

A massa ventricular esquerda (MVE) foi obtida como recomendado pelo método de Penn, segundo a convenção da Sociedade Americana de Ecocardiografia (DEVEREUX \& REICHEK, 1977)

$\mathrm{Na}$ análise estatística, para todas as variáveis estudadas, foi utilizada a correlação com peso corporal e idade. Naquelas variáveis com correlação significativa $(\mathrm{p}<0,05$ e coeficiente de correlação acima de 0,70 ) para peso corporal foi utilizada a 
regressão linear. Em relação ao sexo, foi utilizado o teste $\mathrm{t}$ de student para a comparação dos valores médios entre machos e fêmeas e estabelecido o nível de significância (\%). Como a distribuição da amostra era normal (gaussiana), foi ainda realizada estatística descritiva dos dados (média e desvio padrão).

\section{RESULTADOS E DISCUSSÃO}

Imagens ecocardiográficas bidimensionais satisfatórias foram obtidas nos 60 cães estudados (Figuras 1 e 2), estando qualitativamente similares àquelas descritas para outras raças, conforme relatado por THOMAS (1984), O'GRADY et al. (1986), MILLER et al. (1989), LUSK \& ETTINGER (1990), BAYÓN et al. (1994). Os cães estudados possuíam faixa etária variando entre um e cinco anos de idade, com média de $2,9 \pm 1,21$. O peso variou de 22,0 a $37,2 \mathrm{~kg}$, com média de $30,2 \pm 3,98 \mathrm{~kg}$. Com relação à distribuição dos sexos, a amostra constituiu-se de $48,3 \%$ de fêmeas e $51,7 \%$ de machos.

No modo $\mathrm{M}$, das variáveis estudadas, a dimensão interna do ventrículo direito na diástole (VDd) e dimensão interna do ventrículo esquerdo na diástole (VEd) e na sístole (VEs) obtiveram os maiores índices de correlação com o peso corporal (Tabela 1), como observado também por BOON et al. (1983), LOMBARD (1984), GOODING et al. (1986), JACOBS \& MAHJOOB (1988), SISSON \& SCHAEFFER (1991), CRIPPA et al. (1992) e HANTON et al. (1998). Em relação ao sexo, foi encontrada neste estudo diferença significativa.

Quanto ao VDd (Tabela 1), LOMBARD \& ACKERMAN (1984) e HENIK (1995) afirmaram ser o ecocardiograma modo-M um exame sensível para detectar aumentos no ventrículo direito, especi-

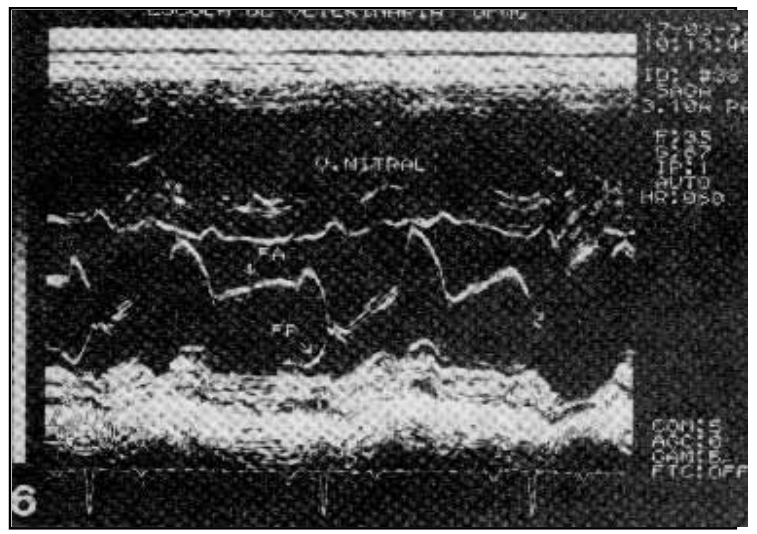

Figura 1 - Modo-M. Traçado da valva mitral com sua cúspide septal (FA) e cúspide parietal (FP) em cães da raça Pastor Alemão do canil da Polícia Militar do Estado de Minas Gerais.

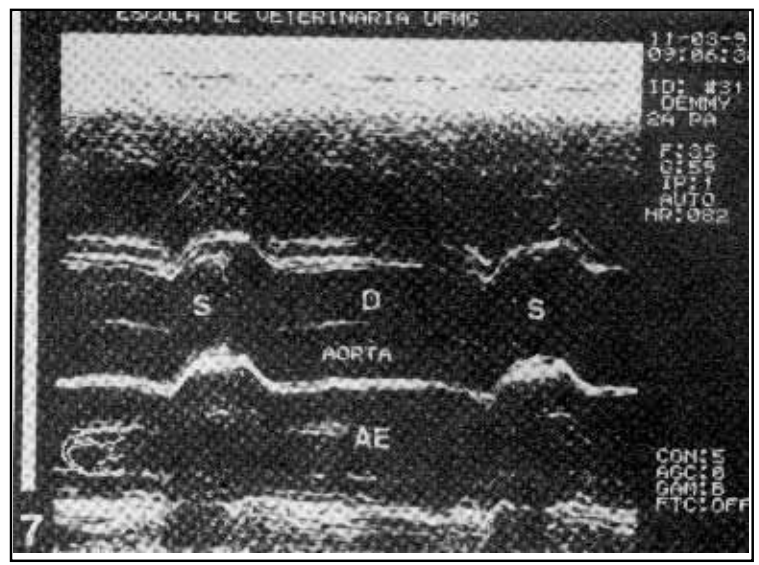

Figura 2 - Modo-M. Traçado da aorta durante o movimento de sístole (S) e diástole (D) em cães da raça Pastor Alemão do canil da Polícia Militar do Estado de Minas Gerais. A aurícula esquerda se encontra ventralmente (AE).

almente em casos de dirofilariose, exemplificando a importância do conhecimento dos valores normais como referência. Em relação ao VEd e VEs, LUSK \& ETTINGER (1990), BÖKENHANS (1992), HENIK (1995) e KOCH et al. (1996) citaram a importância do estabelecimento dos padrões para o diagnóstico não-invasivo de várias doenças, inclusive as cardiomiopatias congestivas, que alteram significativamente essas medidas. PAGE et al. (1993), SNYDER et al. (1995) e KOCH et al. (1996) estudaram essas variáveis em cães de grande porte com a mesma faixa etária e peso corporal, encontrando valores semelhantes aos desta pesquisa.

A massa ventricular esquerda (MVE) demonstrou média de $144,9 \mathrm{~g} \pm 46,7$ e correlação significativa com o peso corporal. GOODING et al. (1986), SISSON \& SCHAEFFER (1991) e PAGE $\boldsymbol{e}$ al. (1993) também encontraram correlação significativa com o peso corporal. PAGE et al. (1993), estudando essa variável em cães da raça Greyhound, observaram valores muito superiores $(287,6 \mathrm{~g} \pm$ 39,1), indicando hipertrofia do coração desses cães, comparados aos demais, mesmo estando fora de treinamento e exercícios regulares há mais de dois anos. Os cães da raça Pastor Alemão deste estudo, embora fossem exercitados regularmente, estavam sem treinamento intenso quando se realizou a pesquisa, não interferindo com os dados encontrados. Em relação ao sexo, houve diferença significativa (Tabela 1), o que também é descrito em seres humanos, como observado por MORCERF (1996). Segundo LOMBARD (1984), GOODING et al. (1986), SISSON \& SCHAEFFER (1991), DROUARDHAELEWYN (1998), a MVE é uma variável com boa aceitação clínica, especialmente no diagnóstico 
Tabela 1 - Índices ecocardiográficos modo-M e suas correlações com sexo e peso corporal em 60 cães da raça Pastor Alemão do canil da Polícia Militar do Estado de Minas Gerais (Belo Horizonte, MG, 1999).

\begin{tabular}{|c|c|c|c|c|c|c|}
\hline \multirow[t]{2}{*}{ Variável $^{(1)}$} & \multirow[t]{2}{*}{$x \pm s^{(*)}$} & \multicolumn{2}{|c|}{$\begin{array}{c}\text { Diferença entre } \\
\text { sexo }\end{array}$} & \multicolumn{3}{|c|}{$\begin{array}{c}\text { Correlação com peso } \\
\text { Corporal }^{(3)}\end{array}$} \\
\hline & & Teste $\mathrm{t}$ & $\mathrm{NS} \%{ }^{(2)}$ & $\mathrm{r}$ & Regressão ${ }^{(4)}$ & $r^{2}$ \\
\hline $\operatorname{VDd}(\mathrm{cm})$ & $1,01 \pm 0,26$ & 4,74 & $<0,01$ & 0,74 & $\mathrm{Y}=-0,43+0,05(\mathrm{~kg})$ & 0,55 \\
\hline $\operatorname{VEd}(\mathrm{cm})$ & $4,17 \pm 0,50$ & 4,48 & $<0,01$ & 0,79 & $\mathrm{Y}=1,18+0,10(\mathrm{~kg})$ & 0,62 \\
\hline $\operatorname{VEs}(\mathrm{cm})$ & $3,10 \pm 0,51$ & 4,47 & $<0,01$ & 0,87 & $\mathrm{Y}=0,29+0,11(\mathrm{~kg})$ & 0,76 \\
\hline MVE(g) & $144,9 \pm 46,7$ & 4,38 & $<0,01$ & 0,77 & $Y=-127+9,0(\mathrm{~kg})$ & 0,59 \\
\hline $\mathrm{AO}(\mathrm{cm})$ & $2,52 \pm 0,16$ & $\mathrm{~ns}$ & - & 0,38 & - & - \\
\hline $\mathrm{AE}(\mathrm{cm})$ & $2,43 \pm 0,21$ & $\mathrm{~ns}$ & - & 0,36 & - & - \\
\hline $\operatorname{SIVd}(\mathrm{cm})$ & $0,96 \pm 0,09$ & $\mathrm{~ns}$ & - & 0,48 & - & - \\
\hline SIVs $(\mathrm{cm})$ & $1,40 \pm 0,09$ & $\mathrm{~ns}$ & - & 0,30 & - & - \\
\hline $\operatorname{PPd}(\mathrm{cm})$ & $0,88 \pm 0,11$ & 3,49 & $<0,01$ & 0,58 & - & - \\
\hline $\operatorname{PPs}(\mathrm{cm})$ & $1,30 \pm 0,12$ & 3,84 & $<0,01$ & 0,51 & - & - \\
\hline SIV/PP & $1,09 \pm 0,15$ & ns & - & ns & - & - \\
\hline $\mathrm{AE} / \mathrm{AO}$ & $0,97 \pm 0,09$ & $\mathrm{~ns}$ & - & $\mathrm{ns}$ & - & - \\
\hline$\%$ ESS & $47,45 \pm 16,2$ & $\mathrm{~ns}$ & - & $\mathrm{ns}$ & - & - \\
\hline$\%$ ЕРP & $49,90 \pm 12,1$ & $\mathrm{~ns}$ & - & $\mathrm{ns}$ & - & - \\
\hline$\% \Delta \mathrm{d}$ & $28,63 \pm 6,52$ & $\mathrm{~ns}$ & - & ns & - & - \\
\hline
\end{tabular}

\footnotetext{
(1) Variável: VDd = dimensão interna do ventrículo direito na diástole, VEd $=$ dimensão interna do ventrículo esquerdo na diástole, VEs = dimensão interna do ventrículo esquerdo na sístole, $\mathrm{MVE}=$ massa ventricular esquerda, $\mathrm{AO}=$ diâmetro da raiz da aorta, $\mathrm{AE}=$ diâmetro interno do átrio esquerdo, $\mathrm{SIVd}=$ espessura do septo interventricular na diástole, SIVs = espessura do septo interventricular na sístole, PPd = espessura da parede posterior do ventrículo esquerdo na diástole, PPs = espessura da parede posterior do ventrículo esquerdo na sístole, SIV/PP = relação espessura do septo interventricular e parede posterior, no final da diástole, $\mathrm{AE} / \mathrm{AO}=$ relação diâmetro do átrio esquerdo e aorta, \%ESS = porcentagem de espessamento sistólico do septo interventricular, \%EPP = porcentagem de espessamento sistólico da parede posterior do ventrículo esquerdo; $\% \Delta \mathrm{d}=$ porcentagem de encurtamento sistólico do diâmetro ventricular esquerdo; ns = não significativo.

${ }^{(2)} \mathrm{NS} \%$ = Nível de significância em porcentagem.

${ }^{(3)}$ Correlação com peso corporal: $\mathrm{P}<0,05$.

${ }^{(4)}$ Regressão: $\mathrm{Y}=$ estimativa da variável dependente para um dado valor de peso

${ }^{(*)} \mathrm{x} \pm \mathrm{s}=$ média \pm desvio padrão

Obs.: Não houve correlação significativa com idade $(\mathrm{P}<0,05)$.
}

de hipertrofia cardíaca, mas a fidelidade da avaliação por esse método pode ser diminuída quando houver evidência de sobrecarga volumétrica do ventrículo esquerdo ou hipertrofia septal assimétrica.

As variáveis diâmetro da raiz da aorta (AO), diâmetro interno do átrio esquerdo (AE), espessura do septo interventricular na diástole (SIVd) e na sístole (SIVs), espessura da parede posterior do ventrículo esquerdo na diástole (PPd) e na sístole (PPs), que se correlacionaram significativamente com o peso corporal, porém com coeficientes de correlação inferiores a 0,58 (Tabela 1) foram também estudadas por LOMBARD (1984), GOODING et al. (1986), JACOBS \& MAHJOOB (1988), CRIPPA et al. (1992) e KOCH et al. (1996), com resultados semelhantes.

$\mathrm{O} \mathrm{AO}$ e o AE (Figura 2) assumem especial importância, pois a sua relação (AE/AO) é um guia prático para detectar aumentos no átrio esquerdo, observados em fase inicial do processo patológico, e que são algumas vezes passados despercebidos por outros métodos complementares, como a radiografia e eletrocardiografia, como relataram BOON et al. (1983), LOMBARD (1984), LOMBARD \& SPENCER (1985) e KOCH et al. (1996).

A SIVd e SIVs obtiveram correlação significativa com o peso corporal, observado por BOON et al. (1983), LOMBARD (1984), GOODING et al. (1986) e SISSON \& SCHAEFFER (1991), que encontraram valores muito semelhantes aos desta pesquisa. BOON et al. (1983), LOMBARD (1984), PAGE et al. (1993) e SNYDER et al. (1995) observaram em cães da raça Greyhound uma hipertrofia do septo interventricular na diástole e na sístole, cujos resultados foram superiores aos encontrados para o Pastor Alemão, e citaram como explicação uma possível variação genética dessa raça em relação às demais. $\mathrm{O}$ mesmo foi observado em relação aos valores de espessura de parede posterior do ventrículo esquerdo na diástole e sístole. BOON et al. (1983), LOMBARD (1984), GOODING et al. (1986) e SISSON \& SCHAEFFER (1991) encontraram valores similares, porém não relataram diferença significativa entre sexos, como foi visto neste experimento (Tabela 1), no qual se estudou uma amostra homogênea em raça, peso corporal e idade.

Tanto as medidas da SIV quanto PP, na diástole e sístole, foram utilizadas para calcular o espessamento sistólico do septo interventricular (\%ESS) e da parede posterior (\%EPP). Para BOON et al. (1983) e KOCH et al. (1996), essas duas variáveis fazem parte dos índices de função ventricular esquerda e são indicadores da contratilidade e complacência do ventrículo esquerdo. Da mesma forma que para BOON et al. (1983), neste estudo não foi encontrada qualquer correlação significativa entre estas variáveis e peso corporal, sexo e idade, porque são índices obtidos a partir de cálculos e resultam em valores constantes, semelhantes para diferentes raças, pesos corporais e idades.

Outra variável estudada foi a relação entre espessura do septo interventricular e parede posteri- 
or do ventrículo esquerdo, ambas na diástole (SIV/PP), cujo valor foi bem próximo ao encontrado por BOON et al. (1983). MARKS (1993) mostrou que na cardiomiopatia hipertrófica essa relação freqüentemente é superior a 1,3 , indicando que o septo é geralmente mais afetado que a parede posterior do ventrículo esquerdo, exemplificando a importância de se conhecer os valores normais dessa variável.

A porcentagem de encurtamento sistólico do diâmetro ventricular esquerdo $(\% \Delta \mathrm{d})$ faz parte dos chamados índices de função ventricular esquerda (MORCERF, 1996). ALLEN (1982), BOON et al. (1983), LOMBARD (1984), SISSON \& SCHAEFFER (1991) e HANTON \& LODOLA (1998) relataram que a \% $\%$ d é um índice preciso da contratilidade e da complacência ventricular esquerda, sendo clinicamente útil para detectar alterações em uma ampla variedade de raças, pesos corporais e idades, pois não tem correlação significativa com esses itens. PAGE $\boldsymbol{e} \boldsymbol{t} \boldsymbol{a l}$. (1993) e SNYDER $\boldsymbol{e t} \boldsymbol{a l}$. (1995) estudando cães da raça Greyhound, e KOCH et al. (1996), estudando raças de grande porte, encontraram valores de $\% \Delta \mathrm{d}$ semelhantes aos destes cães da raça Pastor Alemão (Tabela 1), que, no entanto, estão diferentes dos de outras raças descritas, cujos valores foram maiores, em torno de 30 a $40 \%$ (BOON et al., 1983; LOMBARD, 1984; SISSON \& SCHAEFFER, 1991; KIENLE \& THOMAS, 1995). Uma possível explicação está baseada no porte do animal como foi observado também por SNYDER $\boldsymbol{e t}$ al. (1995) que compararam essa variável em cães da raça Greyhound e obtiveram valores semelhantes.

\section{FONTES DE AQUISIÇÃO}

a - Aparelho de Ultra-som UF 5800A - Fukuda. Tóquio, Japão.

b - Gel para ultra-som UF Cleargel OJ - 20 Fukuda. Tóquio, Japão.

c - Vídeo Grafic Printer Sony UP - 890. Tóquio, Japão.

d - Papel para ultra-som Printing Paper Sony UPP - 110HA Type

IV. Tóquio, Japão.

e - Vídeo-gravador Panasonic, São Paulo, Brasil.

\section{REFERÊNCIAS BIBLIOGRÁFICAS}

ALLEN, D.G. Echocardiography as a research and clinical tool in veterinary medicine. Canadian Veterinary Journal, v.23, n.11, p.313-316, 1982.

BAYÓN, A., PALACIO, M.J.F., MONTES, A. et al. Aspectos ecocardiográficos normales en perros Beagle y Mastín Español en crecimiento. Anales de Veterinaria de Murcia v.9, n.10, p.3-15, 1994

BÖKENHANS, R. Cardiomiopatía dilatada en caninos de raza Dobermann. Revista de Medicina Veterinária (Buenos Aires), v.73, n.4, p.163-167, 1992.
BONAGURA, J.D. Echocardiography. Journal of The American Veterinary Medical Association, v.204, n.4, p.516-522, 1994

BOON, J., WINGFIELD, W.E., MILLER, C.W. Echocardiographic indices in the normal dog. Veterinary Radiology, v.24, n.5, p.214-221, 1983.

CRIPPA, L., FERRO, E., MELLONI, E. et al. Echocardiographic parameters and indices in the normal Beagle dog. Laboratory Animals, v.26, n.3, p.190-195, 1992.

DEVEREUX, R.B., REICHEK, N. Echocardiographic determination of left ventricular mass in man. Circulation, v.55, n.4, p.613-618, 1977.

DROUARD-HAELEWYN, C. Évaluation du fonctionnement myocardique par échographie: réalisation pratique et intérêt chez les carnivores domestiques. Prat Méd Chir Anim Comp, v.33, p. 47-59, 1998.

GOODING, J.P., ROBINSON, W.F., MEWS, G.C Echocardiographic assessment of left ventricular dimensions in clinically normal English Cocker Spaniels. American Journal of Veterinary Research, v.47, n.2, p.296-300, 1986.

HANTON, G., LODOLA, A. Echocardiography, a non-invasive method for the investigation of heart morphology and function in laboratory dogs: 2. effetcs of minoxidil and quinidine on the left ventricle function. Laboratory Animals, v.32, n.2, p.183-190, 1998.

HANTON, G., GEFFRAY, B., LODOLA, A. Echocardiography, a non-invasive method for the investigation of heart morphology and function in laboratory dogs: 1. method and reference values for M-mode parameters. Laboratory Animals, v.32, n.2, p.173-182, 1998.

HENIK, R.A. Echocardiography and doppler ultrasound. In: MILLER, M.S., TILLEY, L.P. (Ed.). Manual of canine and feline cardiology. 2 ed. Philadelphia : Saunders, 1995. p.75-107.

JACOBS, G., MAHJOOB, K. Multiple regression analysis, using body size and cardiac cycle length, in predicting echocardiographic variables in dogs. American Journal of Veterinary Research, v.49, n.8, p.1290-1294, 1988.

JAUDON, J.P., PERROT, C., VIAUD, F. et al. Bases físicas, tecnológicas e semiológicas da ultra-sonografia clínica. A Hora Veterinária, v.11, n.64, p.10-16, 1991.

KIENLE, R.D., THOMAS, W.P. Echocardiography. In: NYLAND, T.G., MATTOON, J.S. (Ed.). Veterinary diagnostic ultrasound. Philadelphia : Saunders, 1995. p.198-256.

KIRBERGER, R.M. Doppler echocardiography: facts and physics for practitioners. Compendium on Continuing Education for the Practicing Veterinarian, v.13, n.11, p.1679-1686, 1991.

KOCH, J., PEDERSEN, H.D., JENSEN, A.L. et al. M-mode echocardiographic diagnosis of dilated cardiomyopathy in giant breed dogs. Journal Veterinary Medical Association, v.43, n.5, p.297-304, 1996.

LOMBARD, C.W. Normal values of the canine M-mode echocardiogram. American Journal of Veterinary Research, v.45, n.10, p.2015-2018, 1984. 
LOMBARD, C.W., ACKERMAN, N. Right heart enlargement in heartworm-infected dogs. Veterinay Radiology, v.25, n.5, p. 210-217, 1984.

LOMBARD, C.W., SPENCER, C.P. Correlation of radiographic, echocardiographic and electrocardiographic signs of left heart enlargement in dogs with mitral regurgitation. Veterinary Radiology, v.26, n.3, p.89-97, 1985.

LUSK. R.H., ETTINGER, S.J. Echocardiographic techniques in the dog and cat. Journal of the American Animal Hospital Association, v.26, n.5, p.473-488, 1990.

MARKS, C.A. Hypertrophic cardiomyopathy in a dog. Journal of the American Veterinary Medical Association, v.203, n.7, p.1020-1022, 1993.

MARTIN, M.W.S. Small animal echocardiography. In: GODDARD, P.J. (Ed). Veterinary ultrasonography. UK Cab International, 1995. p.131-165.

MILLER, M.W., KNAUER, K.W. HERRING, D.S Echocardiography: principles of interpretation. Seminars in Veterinary Medicine and Surgery (Small Animal), v.4, n.1, p.58-76, 1989.

MORCERF, F.A.P. Ecocardiografia uni-bidimensional, transesofágica e doppler. 2 ed. Rio de Janeiro : Revinter, 1996. $671 \mathrm{p}$

O'GRADY, M.R., BONAGURA, J.D., POWERS, J.D. et al.
Quantitative cross-sectional echocardiography in the normal dog. Veterinary Radiology, v.27, n.2, p.34-49, 1986.

PAGE, A., EDMUNDS, G., ATWELL, R.B. Echocardiographic values in the Greyhound. Australian Veterinary Journal, v.70, n.10, p.361-364, 1993

SAHN, D.J., DeMARIA, A., KISSLO, J. et al. Recommendations regarding quantitation in m-mode echocardiography: results of a survey of echocardiographic measurements. Circulation, v.58, n.6, p.1072-1083. 1978.

SISSON, D., SCHAEFFER, D. Changes in linear dimensions of the heart, relative to body weight, as measured by M-mode echocardiography in growing dogs. American Journal of Veterinary Research, v.52, n.10, p.1591-1596, 1991.

SNYDER, P.S., SATO, T., ATKINS, C.E. A comparison of echocardiographic indices of the nonracing, healthy greyhound to reference values from other breeds. Veterinary Radiology \& Ultrasound, v.36, n.5, p.387-392, 1995.

THOMAS, W.P. Two-dimensional, real-time echocardiography in the dog - technique and anatomic validation. Veterinary Radiology, v.25, n.2, p.50-64, 1984.

THOMAS, W.P., GABER, C.E., JACOBS, G.J. $\boldsymbol{e}$ t $\boldsymbol{a l}$. Recommendations for standards in transthoracic twodimensional echocardiography in the dog and cat. The echocardiography Committee of the Specialty of Cardiology, American College of Veterinary Internal Medicine. Journal of Veterinary Internal Medicine, v.7, n.4, p.247-252, 1993.

Ciência Rural, v. 30, n. 5, 2000. 